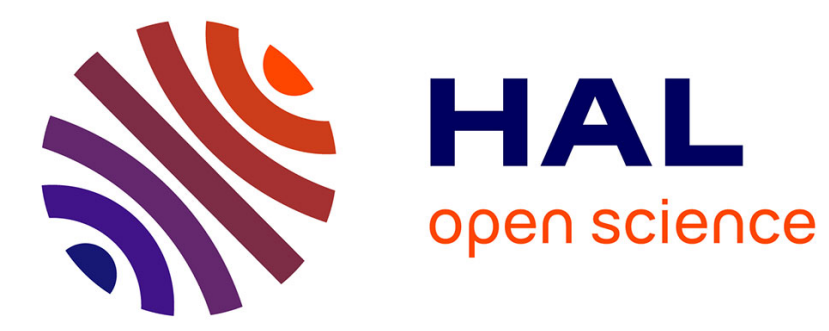

\title{
Various Ways to Compute the Continuous-Discrete Extended Kalman Filter
}

Paul Frogerais, Jean-Jacques Bellanger, Lotfi Senhadji

\section{To cite this version:}

Paul Frogerais, Jean-Jacques Bellanger, Lotfi Senhadji. Various Ways to Compute the ContinuousDiscrete Extended Kalman Filter. IEEE Transactions on Automatic Control, 2012, 57 (4), pp.1000 1004. 10.1109/TAC.2011.2168129 . hal-00904376

\section{HAL Id: hal-00904376 https://hal.science/hal-00904376}

Submitted on 5 Dec 2013

HAL is a multi-disciplinary open access archive for the deposit and dissemination of scientific research documents, whether they are published or not. The documents may come from teaching and research institutions in France or abroad, or from public or private research centers.
L'archive ouverte pluridisciplinaire HAL, est destinée au dépôt et à la diffusion de documents scientifiques de niveau recherche, publiés ou non, émanant des établissements d'enseignement et de recherche français ou étrangers, des laboratoires publics ou privés. 


\title{
Various ways to compute the continuous-discrete Extended Kalman Filter
}

\author{
Paul Frogerais, Jean-Jacques Bellanger and Lotfi Senhadji
}

\begin{abstract}
The Extended Kalman Filter (EKF) is a very popular tool dealing with state estimation. Its continuousdiscrete version (CD-EKF) estimates the state trajectory of continuous-time nonlinear models, whose internal state is described by a stochastic differential equation and which is observed through a noisy nonlinear form of the sampled state. The prediction step of the CD-EKF leads to solve a differential equation that cannot be generally solved in a closed form. This technical note presents an overview of the numerical methods, including recent works, usually implemented to approximate this filter. Comparisons of theses methods on two different nonlinear models are finally presented. The first one is the Van der Pol oscillator which is widely used as a benchmark. The second one is a neuronal population model. This more original model is used to simulate EEG activity of the cortex. Experiments showed better stability properties of implementations for which the positivity of the prediction matrix is guaranteed.
\end{abstract}

Index Terms-Extended Kalman filters, Continuousdiscrete filters, Runge-Kutta method, nonlinear models

\section{INTRODUCTION}

The present study focuses on the way to implement the continuous-discrete Extended Kalman Filter [1] (EKF). This non-linear extension of the Kalman filter turns out pertinent and more appropriate in many circumstances. In fact, EKF is popular in many domains [2] [3] and in some situations it can outperform more sophisticated filters [4]. Moreover, it is often used in benchmarking when a new filtering approach is proposed [5] [6] [7]. To implement this Continuous-Discrete filter (CD-EKF) [1] an intricate design is necessary to avoid instability and ensure good accuracy. Indeed CD-EKF cannot be directly implemented because a system of two ordinary differential equations, called the moment differential equation, must be integrated between two consecutive sampling time instants. Thus, still today researchers

Manuscript received February 16, 2010

P. Frogerais, J.J Bellanger and L. Senhadji are with INSERM, U642, Rennes, F-35000, France; Université de Rennes 1, LTSI, Rennes, F-35000, France (corresponding author: +33 223235 619; e-mail: paul.frogerais@univ-rennes1.fr). propose methods to integrate them efficiently in order to improve its performances [8] [9] [4].

In this paper, after a reminder of the Extended Kalman filter in section II, an overview of recent developments in $\mathrm{CD}-\mathrm{EKF}$ is reported and an original CD-EKF approach is presented and compared with preexisting ones in section III. Two approaches are pointed out. The first one uses deterministic schemes to numerically integrate the moment differential equation in the prediction step of the EKF [8] [4]. The second approach uses stochastic scheme approximation to simulate the continuousdiscrete model with a discrete-discrete one and to apply the discrete-discrete EKF [9] [10]. Finally in the last section, all these methods are compared experimentally on a Van der Pol oscillator and a neuronal population model [11] to investigate their accuracy and stability.

\section{Extended KALman Filtering (EKF)}

\section{A. Discrete-Discrete Extended Kalman Filter}

Nonlinear models considered here are described by:

$$
\begin{aligned}
X_{k} & =f\left(X_{k-1}, W_{k}\right) \\
Y_{k} & =h\left(X_{k}\right)+V_{k}
\end{aligned}
$$

where $W_{k} \sim \mathcal{N}\left(0, Q_{w}\right), V_{k} \sim \mathcal{N}\left(0, Q_{v}\right), k \in \mathbb{Z}$ are mutually independent and where $f: \mathbb{R}^{N_{x}} \mapsto \mathbb{R}^{N_{x}}$ and $h: \mathbb{R}^{N_{x}} \mapsto \mathbb{R}^{N_{y}}$ are nonlinear functions. The discretediscrete EKF (DD-EKF) [1] uses a first order Taylor approximation of the nonlinear state function (1) around the current estimation $\hat{X}_{k-1 \mid k-1}$ of $X_{k-1}$ and around the mean $E\left[W_{k}\right]=0$ of the state noise to obtain the following local approximation:

$$
\begin{aligned}
& X_{k} \simeq \tilde{X}_{k}=f\left(\hat{X}_{k-1 \mid k-1}, 0\right)+ \\
& F_{k}\left(X_{k-1}-\hat{X}_{k-1 \mid k-1}\right)+G_{k} W_{k}
\end{aligned}
$$

where,

$$
F_{k}=\frac{\partial f}{\partial X_{k-1}}\left(\hat{X}_{k-1 \mid k-1}, 0\right)
$$

and

$$
G_{k}=\frac{\partial f}{\partial W_{k}}\left(\hat{X}_{k-1 \mid k-1}, 0\right)
$$


The prediction step of the DD-EKF is then given by:

$$
\begin{aligned}
\hat{X}_{k \mid k-1} & =E\left[\tilde{X}_{k}\right]=f\left(\hat{X}_{k-1 \mid k-1}, 0\right) \\
P_{k \mid k-1} & =E\left[\tilde{X}_{k} \tilde{X}_{k}^{T}\right] \\
& =F_{k} P_{k-1 \mid k-1} F_{k}^{T}+G_{k} Q_{W} G_{k}^{T}
\end{aligned}
$$

The correction step obeys to:

$$
\begin{aligned}
K_{k} & =P_{k \mid k-1} H_{k}^{T}\left(H_{k} P_{k \mid k-1} H_{k}^{T}+Q_{v}\right)^{-1} \\
\hat{X}_{k \mid k} & =\hat{X}_{k \mid k-1}+K_{k}\left(Y_{k}-h\left(\hat{X}_{k \mid k-1}\right)\right) \\
P_{k \mid k} & =P_{k \mid k-1}-K_{k} H_{k} P_{k \mid k-1}
\end{aligned}
$$

where $H_{k}$ corresponds to the linearization of the observation function $h($.$) around the prediction \hat{X}_{k \mid k-1}$ :

$$
H_{k}=\frac{\partial h}{\partial X_{k}}\left(\hat{X}_{k \mid k-1}\right)
$$

\section{B. Continuous-Discrete Extended Kalman Filter}

The Markov models evolution considered here will be described by the following nonlinear stochastic differential equation (SDE):

$$
d X=f(X, t) d t+G(t) d \beta
$$

where $X(t) \in \mathbb{R}^{N_{x}}, f$ is a nonlinear function, $G(t)$ is a $N_{x} \times N_{w}$ matrix and $\{\beta(t), t>0\}$ is a Brownian process with diffusion matrix $Q(t)$. The observation follows equation (2) with $X_{k}=X\left(t_{k}\right)$ and the sampling period $\Delta_{y}=t_{k}-t_{k-1}$ is a constant. The prediction step of the continuous-discrete EKF (CD-EKF) consists in integrating from $t_{k-1}$ to $t_{k}$ a system of two coupled ordinary differential equations [1] called differential moment equation in [8]:

$$
\begin{aligned}
\frac{d m}{d t} & =f(m, t) \\
\frac{d P}{d t} & =R(P, t) \\
& =F(m, t) P+P F(m, t)^{T}+G(t) Q(t) G(t)^{T}
\end{aligned}
$$

with $m\left(t_{k-1}\right)=\hat{X}_{k-1 \mid k-1}$ and $P\left(t_{k-1}\right)=P_{k-1 \mid k-1}$ where $F(m, t)=\frac{\partial f}{\partial X}(m, t)$. Then we obtain the prediction mean $\hat{X}_{k \mid k-1}=m\left(t_{k}\right)$ and covariance $P_{k \mid k-1}=$ $P\left(t_{k}\right)$. The correction step is the same as in the discretediscrete EKF, equation (5).

\section{NUMERICAL APPROXIMATION METHODS}

Two methodologies to approximate the CD-EKF in general cases are presented. The first one uses deterministic scheme to integrate the differential moment equation (7)(8) and the second one introduces a discretediscrete EKF filter on a discrete version of the stochastic differential equation (6).
TABLE I

RUNGE-KUTTA OPERATOR $\mathfrak{R} \mathfrak{K}_{i}^{\delta}$ ON A FUNCTION $f: \mathbb{R}^{N_{x}} \times \mathbb{R} \mapsto \mathbb{R}^{N_{x}}$

\begin{tabular}{lc}
\hline $\begin{array}{l}\text { Euler } \\
(i=1)\end{array}$ & $\left(\mathfrak{R} \mathfrak{K}_{1}^{\delta} f\right)(X, t)=\delta f(X, t)$ \\
Heun & $\left(\mathfrak{R} \mathfrak{K}_{2}^{\delta} f\right)(X, t)=\frac{\delta}{2}(K 1+K 2)$ \\
$(i=2)$ & $K 1=f(X, t)$ \\
& $K 2=f(X+\delta K 1, t+\delta)$ \\
RK4 & $\left(\mathfrak{R} \mathfrak{K}_{4}^{\delta} f\right)(X, t)=\frac{\delta}{6}(K 1+2 K 2+2 K 3+K 4)$ \\
$(i=4)$ & $K 1=f(X, t)$ \\
& $K 2=f\left(X+\frac{\delta}{2} K 1, t+\frac{\delta}{2}\right)$ \\
& $K 3=f\left(X+\frac{\delta}{2} K 2, t+\frac{\delta}{2}\right)$ \\
& $K 4=f(X+\delta K 3, t)$ \\
\hline
\end{tabular}

A. Numerical integration of the differential moment equation

A natural way is to use numerical integration scheme [8] [4] to solve numerically (7) and (8) on $\left[t_{k-1}, t_{k}\right]$. By introducing intermediate time steps $t_{k, 1}<\ldots<$ $t_{k, n}<\ldots<t_{k, \alpha}$, where $t_{k, 1}=t_{k-1}, t_{k, \alpha}=t_{k}$ and $\delta=t_{k, n}-t_{k, n-1}$ is a constant, the mean $m\left(t_{k}+n \delta\right)$ and the covariance $P\left(t_{k}+n \delta\right)$ can be recursively approximated by a i-th order explicit Runge-Kutta method:

$$
\begin{aligned}
m_{k, n} & =m_{k, n-1}+\left(\mathfrak{R} \mathfrak{K}_{i}^{\delta} f\right)\left(m_{k, n-1}, t_{k, n-1}\right) \\
P_{k, n} & =P_{k, n-1}+\left(\mathfrak{R} \mathfrak{K}_{i}^{\delta} R\right)\left(P_{k, n-1}, t_{k, n-1}\right)
\end{aligned}
$$

where $\mathfrak{R} \mathfrak{K}_{i}^{\delta} f(X, t)$ is a i-th order Runge-Kutta operator defined in the table I.

Unfortunately, for large $\delta$ the stability of these schemes is not ensured. Furthermore approximated solution of the covariance can lead to a non-positive semidefinite matrix and this last property is essential to guarantee the convergence of the EKF. That is why in [8] Mazzoni proposes a Taylor Heun approximation scheme for the mean equation (7) and a modified GaussLegendre scheme for the covariance equation (8). Both schemes are A-stable and consistent with order $\mathcal{O}\left(\delta^{2}\right)$ [8]. Furthermore the Gauss Legendre scheme was specially developed to ensure the semi-positivity of the prediction error covariance matrix.

To guarantee this semi-positivity, another convenient approach is used in [4]. The matrix $P_{k \mid k-1}$ can also be obtained by integrating from $t_{k-1}$ to $t_{k}$ the differential equation which governs the fundamental matrix $\phi$ corresponding to system (7) after linearization:

$$
\frac{d \phi(t)}{d t}=F(m, t) \phi(t)
$$

where $F=\frac{\partial f}{\partial X}, m(t)$ fulfills equation (7) and $\phi\left(t_{k-1}\right)=$ $I$ where $I$ is the identity matrix. The prediction error 
covariance matrix is then:

$$
\begin{aligned}
P_{k \mid k-1} & =\phi\left(t_{k}\right) P_{k-1 \mid k-1} \phi\left(t_{k}\right)^{T} \\
& +\int_{t_{k-1}}^{t_{k}} \phi(t) G(t) Q G(t)^{T} \phi(t)^{T} d t
\end{aligned}
$$

Then, classical numerical schemes can be used for the integration of (7) and (10) and the integral in the covariance equation (11) can be approximated by quadrature. This leads to a semi-positive definite approximation of the prediction covariance matrix. This formulation is used in [4] where authors introduce a fourth order Runge-Kutta scheme to solve (10) and (7).

\section{B. Discrete approximation of the $S D E$}

Another original approach, proposed in this section, consists in using Stochastic Runge-Kutta schemes [12] [10] to approximate the process $\left\{X(t), t_{k-1} \leq t \leq t_{k}\right\}$ described by the SDE (6) at discrete instants of time $t_{k, 1}<\ldots<t_{k, n}<\ldots<t_{k, \alpha}$ where $t_{k, 0}=t_{k-1}, t_{k, \alpha}=$ $t_{k}$ and $\delta=t_{k, n}-t_{k, n-1}$ :

$$
\begin{aligned}
& X_{k, n}=X_{k, n-1}+\left(\mathfrak{R} \mathfrak{K}_{i}^{\delta} f_{w_{k, n}}^{\delta}\right)\left(X_{k, n-1}, t_{k, n-1}\right) \\
& X_{k, n}=f_{r k i}^{\delta}\left(X_{k, n-1}, w_{k, n}\right)
\end{aligned}
$$

with $w_{k, n} \sim \mathcal{N}(0, Q \delta)$ and

$$
f_{w}^{\delta}(X, t)=f(X, t)+\frac{G(t)}{\delta} w
$$

Then we can rewrite $X_{k}=X_{k, \alpha} \simeq X\left(t_{k}\right)$ from $X_{k-1}=$ $X_{k, 0} \simeq X\left(t_{k-1}\right)$ and $W_{k}=\left[w_{k, 1}, \ldots, w_{k, \alpha}\right]$, as:

$$
X_{k}=g_{r k i}^{\delta}\left(X_{k-1}, W_{k}\right)
$$

where $g_{r k i}^{\delta}$ is a composite function:

$$
\begin{array}{r}
g_{r k i}^{\delta}\left(X_{k-1}, W_{k}\right)=f_{r k i}^{\delta}\left(f_{r k i}^{\delta}(\ldots\right. \\
\left.\left.f_{r k i}^{\delta}\left(f_{r k i}^{\delta}\left(X_{k-1}, w_{k, 1}\right), w_{k, 2}\right) \ldots\right), w_{k, \alpha}\right)
\end{array}
$$

Then we obtain a discrete approximation (14) of the SDE (6) by a i-th order stochastic Runge-Kutta scheme which has the same form as a nonlinear discrete-discrete model, equations (1) and (2). The DD-EKF needs then to calculate the Jacobian functions $G X_{r k i}^{\delta}=\frac{\partial g_{r k i}^{\delta}}{\partial X}$ and $G W_{r k i}^{\delta}=\frac{\partial g_{r k i}^{\delta}}{\partial W}$. If we know the Jacobian functions of the i-th order stochastic Runge-Kutta scheme $F X_{r k i}^{\delta}=\frac{\partial f_{r k i}^{\delta}}{\partial X}$ and $F w_{r k i}^{\delta}=\frac{\partial f_{r k i}^{\delta}}{\partial w}$ (see appendix), they follow the form:

$$
\begin{array}{r}
G X_{r k i}^{\delta}\left(X_{k-1}, W_{k}\right)=\prod_{n=1}^{\alpha} F X_{r k i}^{\delta}\left(X_{k, n-1}, w_{k, n}\right) \\
G W_{r k i}^{\delta}\left(X_{k-1}, W_{k}\right)=\left[G w_{r k i, 1}^{\delta}\left(X_{k, 0}, w_{k, 1}\right) \mid\right. \\
\left.G w_{r k i, 2}^{\delta}\left(X_{k, 1}, w_{k, 2}\right)|\ldots| G w_{r k i, \alpha}^{\delta}\left(X_{k, \alpha-1}, w_{k, \alpha}\right)\right]
\end{array}
$$

where for $j=1, \ldots, \alpha-1$

$$
\begin{aligned}
& G w_{r k i, j}^{\delta}\left(X_{k, j-1}, w_{k, j}\right)=\frac{\partial g_{r k i}^{\delta}}{\partial w_{k, j}}\left(X_{k, j-1}, w_{k, j}\right)= \\
& \prod_{n=0}^{\alpha-j-1} F X_{r k i}^{\delta}\left(X_{k, \alpha-n-1}, w_{k, \alpha-n}\right) F w_{r k i}^{\delta}\left(X_{k, j-1}, w_{k, j}\right)
\end{aligned}
$$

and for $j=\alpha$ :

$$
G w_{r k i, \alpha}^{\delta}\left(X_{k, \alpha-1}, w_{k, \alpha}\right)=F w_{r k i}^{\delta}\left(X_{k, \alpha-1}, w_{k, \alpha}\right)
$$

An interesting property of this second way to compute the CD-EKF is that it ensures the semi-definite positivity of $P_{k \mid k-1}$, see (4). In [9], the author proposes a similar approach but with an ordinary stochastic Euler approximation of the SDE which correspond to let $i=1$. The extension to higher order stochastic Runge-Kutta schemes $(i=2, i=4)$ proposed here improves stability and precision of the filter. However, explicit Runge-Kutta schemes are not stochastically A-stable [13] and a priori can lead to unstable solution for large $\delta$, whereas in [8] deterministic A-stability is ensured.

\section{Performed implementations}

From these two main methodologies, five approximated CD-EKF named here MC-THGL [8], MC-RK4, MF-RK4 [4], D-EULER [9] and D-SRK4 (proposed here) have been implemented in the experimental section. These implementations written in $\mathrm{C}++$ programming language use BFilt [14] which is an open-source library on Bayesian filtering (Kalman, EKF, UKF, particle filter, ...). Main features of these methods are summarized in the table II. MC-RK4 and MC-THGL methods numerically integrate the differential equation of the Mean (7) and the Covariance prediction error (8). A RK4 scheme is used for the first one. Note that this intuitive CD-EKF implementation, proposed in [15] does not guarantee the positivity of the covariance $P_{k \mid k-1}$ when the step size is too large. For the second one [8], a Taylor Heun approximation is used for the mean and a modified Gauss-Legendre scheme for the covariance. This scheme guarantees A-stability and semi-positivity of $P_{k \mid k-1}$. The MF-RK4 [4] integrates the Mean (7) and the Fundamental matrix (10) by a RK4 method and thus also ensures the semi-positivity of $P_{k \mid k-1}$. The DEuler and D-SRK4 methods use discretization method of the SDE by respectively a stochastic Euler scheme and a fourth order Stochastic Runge-Kutta (SRK4) scheme. The D-Euler method is equivalent to the method implemented in [9]. 
TABLE II

FEATURES OF THE DIFFERENT IMPLEMENTATIONS OF THE CD-EKF TESTED IN THE SECTION IV-B

\begin{tabular}{|c|c|c|c|c|c|c|}
\hline \multirow{2}{*}{$\begin{array}{l}\text { Implementation } \\
\text { methods }\end{array}$} & \multicolumn{2}{|c|}{ Numerical integration } & \multirow{2}{*}{$\begin{array}{l}\text { Discrete Approximation } \\
\text { of the SDE (6) }\end{array}$} & \multicolumn{2}{|c|}{ Stable properties } & \multirow{2}{*}{ References } \\
\hline & of (7) and (8) & of (7) and (10) & & A-Stable & $P_{k \mid k-1}>0$ & \\
\hline MC-THGL & $*$ & & & $*$ & $*$ & [8] \\
\hline MC-RK4 & $*$ & & & & & [15] \\
\hline MF-RK4 & & * & & & $*$ & {$[4]$} \\
\hline D-EULER & & & $*$ & & $*$ & [9] \\
\hline D-SRK4 & & & $*$ & & * & proposed here \\
\hline
\end{tabular}

\section{ApplicAtions}

CD-EKF of a Van der Pol oscillator and a neuronal population model used to model EEG activity is presented in this section. These models are described in section IV-A. In section IV-B, the different ways to compute the CD-EKF (table II) are implemented and compared for each model.

\section{A. Models}

1) The Van der Pol oscillator: This oscillator, which produces sustained oscillation, is a classical benchmark for numerical computation [16] [8]. It obeys the following stochastic differential equation:

$$
d\left[\begin{array}{l}
v \\
u
\end{array}\right]=\left[\begin{array}{c}
u \\
\lambda\left(1-v^{2}\right) u-v
\end{array}\right] d t+\left[\begin{array}{l}
0 \\
1
\end{array}\right] d \beta(t)
$$

where $\lambda=3$ and initial conditions $X_{0}=[0.5,0.5]^{T}$ and $P_{0}=\operatorname{diag}[0,0.1]$. The observation $Y_{k}$ is a noisy measure of the voltage $v$ at discrete instants of time:

$$
Y_{k}=v\left(t_{k}\right)+V_{k}
$$

where $V_{k} \sim \mathcal{N}\left(0, \sigma_{v}\right)$ is the white measurement noise.

2) The Jansen model: The neuronal population model (Fig. 1) is made up of three sub-populations $P e_{1}, P e_{2}$, $P i$. They represent excitatory pyramidal cells $\left(P e_{1}, P e_{2}\right)$ and inhibitory sub-population $P i$. $P e_{1}$ excites $P e_{2}$ and $P i, P i$ inhibits $P e_{1}$ and $P e_{2}$ excites $P e_{1}$. Each subpopulation connects to another through a connectivity constant $C_{i}$ (mean number of synaptic contacts) supposed known and time invariant [11]. The nonlinear function $S($.$) describes saturation and threshold effects$ in the soma and converts the (mean) membrane potential into a (mean) firing rate of action potentials. Two synaptic linear dynamics are distinguished for the two respective sub-populations types, each represented by a Laplace transfer function: $h(s)=\frac{\alpha}{(\alpha+s)^{2}}$, where $\alpha \in\{a, b\}$. Time constant values $1 / a$ and $1 / b$ are fixed in accordance with the literature [11]. The influence of the cortical neighborhood is described by a continuoustime Gaussian white noise $W(t)$ with a positive mean $m_{w}$ and a diffusion coefficient $\sigma$. Synaptic gains $A, B$

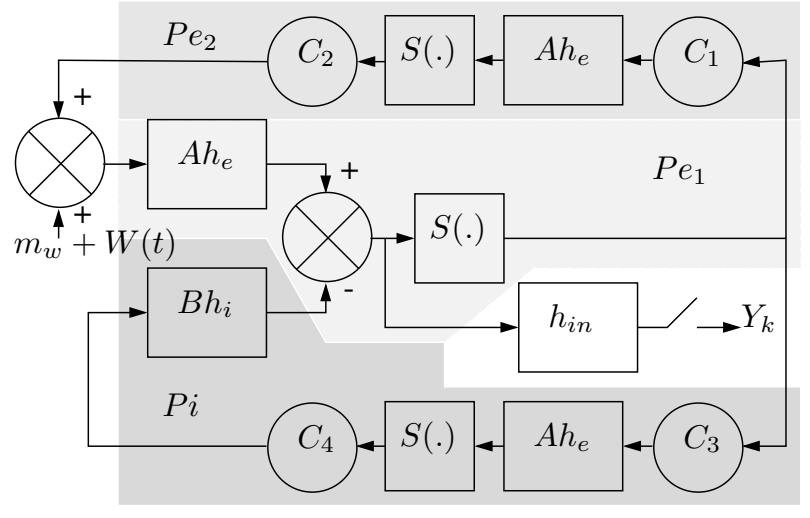

Fig. 1. The jansen model

from respectively excitatory and inhibitory cells are fixed here to $A=5$ and $B=30$. The gain $G P H$, the high pass filter transfer function $h_{p h}$ and the sampler (Fig. 1) represent the instrumentation device used. The signal recorded corresponds to the mean membrane potential of the pyramidal cells $P e$. This model can be expressed by the following stochastic differential equation:

$$
d X=f(X) d t+G d \beta_{t}
$$

where $X \in \mathbb{R}^{7}$ is the state vector, $G$ is a constant diffusion vector and $f$ is the nonlinear drift (it contains $S($.$) and h$ functions). $\left\{\beta_{t}, t>0\right\}$ is a scalar Brownian motion process where $\beta_{t}-\beta_{\tau} \sim \mathcal{N}(0, t-\tau)$ with $t>\tau$. Gaussian white noise $W(t)=G d \beta_{t} / d t$ corresponds to a formal derivative of the Brownian process $\beta_{t}$. The influence of the input mean $m_{w}$ is included in $f(X)$. The output signal $Y_{k}$ is a noisy linear form of the state $X(t)$ at instants $t=t_{k}$ :

$$
Y_{k}=H X\left(t_{k}\right)+v_{k}
$$

with an additive white instrumental noise $v_{k} \sim \mathcal{N}\left(0, \sigma_{v}\right)$.

\section{B. EKF Filtering}

The five approximated CD-EKF presented in section III-C, were implemented for each model. Main features of these methods are reported in table II. In the sequel, we explore the stability of each implementation for 
Van Der Pol

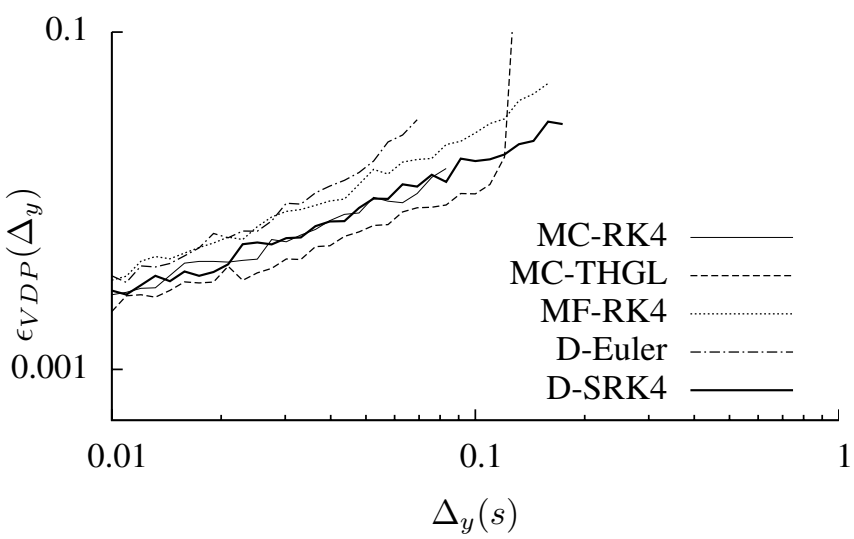

Fig. 2. Estimation error of the different CD-EKF approximation for the Van Der Pol model

different values of the sampling period $\Delta_{y}$ where $\alpha=1$. For each model $M \in\{V D P=\operatorname{Van} \operatorname{der}$ Pol, $J=$ Jansen $\}$ several sets $\left\{\left(Y_{1: N, \Delta_{y}}^{i}, X_{1: N, \Delta_{y}}^{i}\right), i=1, \ldots, N_{\beta}\right\}$ of state trajectories and observation signals were simulated numerically (Stochastic Runge Kutta 4) with different Brownian realizations $\left\{\beta^{i}(t), 0<t<T\right\}$, $i=1, \ldots, N_{\beta}$, each with a very small increment time to obtain weak error relatively to time continuous trajectory, and different values of the sampling period $\Delta_{y}=t_{k}-t_{k-1}$. For each $M$, SRK4 method was used with a simulation step $\delta_{s}$ kept sufficiently large. Then for each couple $\left(Y_{1: N, \Delta_{y}}^{i}, X_{1: N, \Delta_{y}}^{i}\right)$ an estimation $\hat{X}_{1: N, \Delta_{y}}^{i}$ of the corresponding state trajectory was performed by each implemented method MC-THGL, MC-RK4, MFRK4, D-EULER and D-SRK4. A distance $\epsilon_{M}^{i}\left(\Delta_{y}\right)=$ $l_{M}\left(S_{1: N, \Delta_{y}}^{i}, \hat{S}_{1: N, \Delta_{y}}^{i}\right)$ was then evaluated by calculating the sum on a set of selected state variables of the mean square errors between the true simulated trajectories and the estimated ones. For the Van der Pol oscillator the selected state coordinate correspond to the voltage $S_{1: N, \Delta_{y}}=v_{1: N}$. For the Jansen model the selected variables are the three mean membrane potentials.

Results are reported in Fig. 2 and 3. For each implemented method, $\epsilon_{M}\left(\Delta_{y}\right)$ is plotted for different values of the sampling period $\Delta_{y}$ which here is always equal to the step size of the integration methods $\delta(\alpha=1)$. Note that when $\Delta_{y}$ becomes larger, errors due to the integration method appear and can affect the stability of the filter. On Fig. 2 and 3, some curves stop before reaching the right side illustrating that the corresponding implemented CD-EKF becomes suddenly unstable for higher values.

For the Van der Pol model (Fig. 2), all filters failed for $\Delta_{y}>0.18 s$. For all values, MF-RK4 and the proposed method: D-SRK4 have a larger stability range. However
Jansen

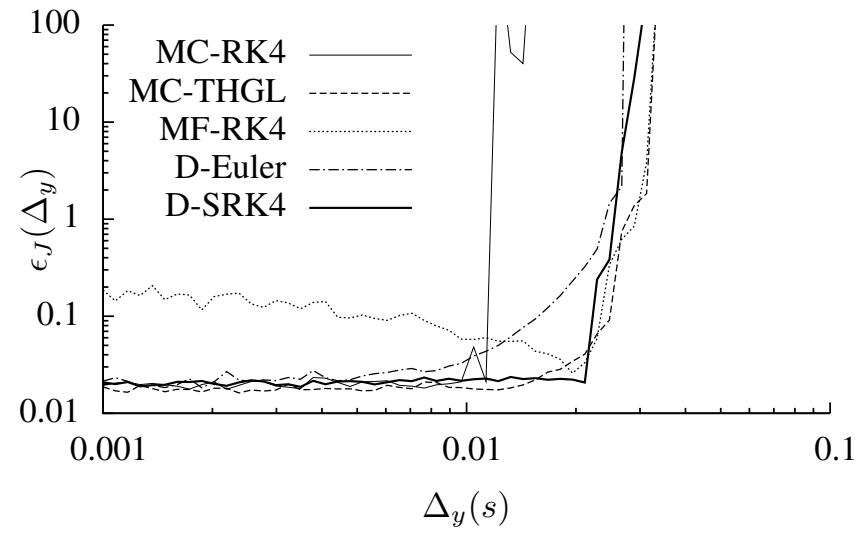

Fig. 3. Estimation error of the different CD-EKF approximation for the Jansen model

MF-RK4 and D-Euler lead, on their stability ranges, to larger errors. For the Jansen model (Fig. 3), MFRK4, MC-THGL and D-SRK4 implementations have large stability ranges. For $\Delta_{y}<0.01 s$, estimation error values are quite similar for all methods except for the MF-RK4 where they are bigger. Globally, MF-RK4, MC-THGL and D-SRK4 implementations give the best performances in stability and estimation error. Results show that MC-RK4 method (the only case where the positivity of $P_{k \mid k-1}$ is not guaranteed), is stable over a smaller range of $\Delta_{y}$ values than other implementations.

\section{DISCUSSION}

Different ways to implement the CD-EKF were presented and evaluated on a classical benchmark model and on a more original model simulating EEG activity. In [9] authors proposed to discretize the stochastic differential equation with the stochastic Euler scheme. Here, an extension to higher order stochastic Runge-Kutta schemes is proposed to enlarge discretization step intervals in which the stability is ensured. To our knowledge, this extension has never been proposed. Other implementations were also investigated and experimentally compared with this one. Results pointed out that the proposed D-SRK4 approach is only slightly more expensive in terms of computation than methods proposed by [4] [9] [8] and furthermore can be more stable for the two models. Experiments confirmed also that when schemes guarantee the semi-positivity of $P_{k \mid k-1}$ implementations give better stability results. The discretization methodology of the stochastic differential equation (reported in section III-B) to compute numerically the continuous-discrete extended Kalman filter can obviously be extended to other filtering methods as the unscented Kalman filtering, 
particle filtering, divided difference filters (DD1 and DD2).

\section{APPENDIX}

Here the Jacobian functions $F X_{r k i}^{\delta}=\frac{\partial f_{r k i}^{\delta}}{\partial X}$ and $F w_{r k i}^{\delta}=\frac{\partial f_{r k i}^{\delta}}{\partial w}$ are expressed for a discrete Runge-Kutta scheme (12) from the Jacobian function $J_{X}(x, t)=$ $\frac{\partial f}{\partial X}(x, t)$ of the drift term of the SDE (6) and $J_{w}(t)=$ $G(t) / \delta$ where $G(t)$ is the diffusion term of (6) with $\mathrm{i}=1$ (Euler):

$$
\begin{aligned}
& F X_{r k 1}^{\delta}\left(X, w_{n}\right)=I+J_{X}(X, t) \delta \\
& F w_{r k 1}^{\delta}\left(X, w_{n}\right)=J_{w}(t) \delta
\end{aligned}
$$

$i=2$ (Heun):

$$
\begin{aligned}
& K 1=f_{w}^{\delta}\left(X_{k-1}, t_{k-1}\right) \\
& K 2=f_{w}^{\delta}\left(X_{k-1}+K 1 \delta, t_{k}\right) \\
& J 1_{X}=J_{X}\left(X_{k-1}, t_{k-1}\right) \\
& J 2_{X}=J_{X}\left(X_{k-1}+K 1 \delta, t_{k}\right)\left(I+\delta J 1_{X}\right) \\
& J 1_{w}=J_{w}\left(t_{k-1}\right) \\
& J 2_{w}=J_{X}\left(X_{k-1}+K 1 \delta, t_{k}\right)\left(\delta J 1_{w}\right) \\
& F X_{r k 2}^{\delta}\left(X, w_{n}\right)=I+\frac{1}{2}\left(J 1_{X}+J 2_{X}\right) \delta \\
& F w_{r k 2}^{\delta}\left(X, w_{n}\right)=\frac{1}{2}\left(J 1_{w}+J 2_{w}\right) \delta
\end{aligned}
$$

and $i=4(\mathrm{SRK} 4)$ :

$$
\begin{aligned}
K 1 & =f_{w}^{\delta}\left(X_{k-1}, t_{k}\right) \\
K 2 & =f_{w}^{\delta}\left(X_{k-1}+\frac{1}{2} K 1 \delta, t_{k-1}+\frac{1}{2} \delta\right) \\
K 3 & =f_{w}^{\delta}\left(X_{k-1}+\frac{1}{2} K 2 \delta, t_{k-1}+\frac{1}{2} \delta\right) \\
K 4 & =f_{w}^{\delta}\left(X_{k-1}+K 3 \delta, t_{k}\right) \\
J 1_{X} & =J_{X}\left(X_{k-1}, t_{k-1}\right) \\
J 2_{X} & =J_{X}\left(X_{k-1}+\frac{1}{2} K 1 \delta, t_{k-1}+\frac{1}{2} \delta\right)\left(I+\frac{1}{2} \delta J 1_{X}\right) \\
J 3_{X} & =J_{X}\left(X_{k-1}+\frac{1}{2} K 2 \delta, t_{k-1}+\frac{1}{2} \delta\right)\left(I+\frac{1}{2} \delta J 2_{X}\right) \\
J 4_{X} & =J_{X}\left(X_{k-1}+K 3 \delta, t_{k}\right)\left(I+\delta J 3_{X}\right) \\
J 1_{w} & =J_{w}\left(t_{k-1}\right) \\
J 2_{w} & =J_{X}\left(X_{k-1}+\frac{1}{2} K 1 \delta, t_{k-1}+\frac{1}{2} \delta\right)\left(\frac{1}{2} \delta J 1_{w}\right) \\
J 3_{w} & =J_{X}\left(X_{k-1}+\frac{1}{2} K 2 \delta, t_{k-1}+\frac{1}{2} \delta\right)\left(\frac{1}{2} \delta J 2_{w}\right) \\
J 4_{w} & =J_{X}\left(X_{k-1}+K 3 \delta, t_{k}\right)\left(\delta J 3_{w}\right)
\end{aligned}
$$

$F X_{r k 4}^{\delta}\left(X, w_{n}\right)=I+\frac{1}{2}\left(J 1_{X}+2 J 2_{X}+2 J 3_{X}+J 4_{X}\right) \delta$

$$
F w_{r k 4}^{\delta}\left(X, w_{n}\right)=\frac{1}{2}\left(J 1_{w}+2 J 2_{w}+2 J 3_{w}+J 4_{w}\right) \delta
$$

\section{REFERENCES}

[1] A. H. Jazwinski, Stochastic processes and filtering theory. Academic Press, Apr. 1970.

[2] P. Ronstan, R. Théoret, and A. E. Moussadek, "Forecasting the interest-rate term structure: Using the model of Fong and Vasicek, the extended Kalman filter and the Bollinger bands," Audencia - Nantes Ecole de Management, Tech. Rep., 2004.

[3] M. Hilairet, F. Auger, and E. Berthelot, "Speed and rotor flux estimation of induction machines using a two-stage extended Kalman filter," Automatica, vol. 45, pp. 1819-1827, 2009.

[4] J. Joseph and J. LaViola, "A comparison of unscented and extended Kalman filtering for estimating quaternion motion," in Proceedings of the American Control Conference, Jun. 2003, pp. 2435-2440.

[5] M. Norgaard, N. K. Poulsen, and O. Ravn, "New developments in state estimation for nonlinear systems," Automatica, vol. 36, no. $11,2000$.

[6] J. S. S. J. Julier and J. K. Uhlmann, "New extension of the Kalman filter to nonlinear systems," in Proc. SPIE Conf. on Signal Processing, Sensor Fusion, and Target Recognition VI, vol. 3068, Orlando, Apr. 1997, pp. 182-193.

[7] N. Gordon, D. Salmond, and A. Smith, "Novel approach to nonlinear/non-Gaussian Bayesian state estiamtion," Radar and Signal Processing, IEE Proceedings F, vol. 140, pp. 107-113, Apr. 1993.

[8] T. Mazzoni, "Computational aspects of continuous-discrete extended Kalman-filtering," Computational Statistics, vol. 23, no. 4, pp. 519-539, 2007.

[9] H. Singer, "Continuous-discrete unscented Kalman filtering," FernUniversitat in Hagen, Tech. Rep. 384, 2005.

[10] J. Wilkie, "Numerical methods for stochastic differential equations," Physical Review E, vol. 70, no. 1, 2004.

[11] B. H. Jansen and V. G. Rit, "Electroencephalogram and visual evoked potential generation in a mathematical model of coupled cortical columns," Biological cybernetics, vol. 73, no. 4, pp. 357-366, 1995.

[12] P. E. Kloeden and E. Platen, Numerical Solution of Stochastic Differential Equations (Stochastic Modelling and Applied Probability). Berlin: Springer, November 2000.

[13] D. Hernandez and R. Spigler, "A-stability of Runge-Kutta methods for systems with additive noise," BIT Numerical Mathematics, vol. 32, pp. 620-633, 1992.

[14] P. Frogerais and J.-J. Bellanger. (2009) A C++ library on Bayesian filtering : BFilt. [Online]. Available: http://paul.frogerais.free.fr

[15] D. Simon, Optimal State Estimation: Kalman, H Infinity, and Nonlinear Approaches. Wiley-Interscience, 2006.

[16] A. Sitz, U. Swartz, J. Kurtz, and U. Voss, "Estimation of parameters and unobserved components for nonlinear systems from noisy time series," Physical Review E, vol. 66, 2002. 